JICA

26,1

\title{
Editorial
}

\section{Making sense of integration}

There has long been an agreement amongst scholars on what integration aims to address. This is to overcome the organisational, professional and regulatory boundaries within the health and social care sector. There has also been a degree of consensus on the reasons why we should embark on care integration. It is hoped that integrating health and social care increases the quality of care for patients by providing person-centred care, ultimately improving care outcomes for everyone. The concept of the triple and indeed quadruple aim of health care has helped to further consolidate thinking of expected outcomes. There is however less agreement about whether or not establishing integrated care solutions for the care sector will lead to cost savings in the short or long term.

Over the last decade, governments have largely supported pilot programmes of care integration without prescribing any particular model of implementation, but hope they will result in increased efficiency. Looking back over the last momentous years, the integration field as a whole has sometimes seemed to struggle to find a way to systematically appraise the diversity in approaches in implementation. Evidence is improving albeit slowly that cost savings may only be possible in the long term.

Integration in health and social care is developing into a field of more systematic inquiry and making its way into medical and nursing curricula in many countries. To underpin this, practitioners and academics need to reconcile the thrust towards variance and diversity in integration programmes with the need for generalisable evidence and transferable learning points for everyone involved in implementing and evaluating integration programmes. Just as integration programmes have few of the hallmarks of systematic policy development and policy implementation; neither does the bulk of the existing evidence at present in and around integration in health and social care offer the key learning points necessary for the rapid diffusion of good practice in integration implementation and evaluation.

This state of affairs is likely to give policy makers little confidence that care integration is underpinned by much else than an arsenal of strong, emotively laden, terms and well-intentioned impetus to work together. The current issue of this journal makes important contributions to this emerging picture of diversity and variability. It reflects neatly the enormous disciplinary scope integration studies have gained as well as the wide range of applications for integration across health and social care fields.

In the first article of the issue, Mark Gaines and Patricia Commiskey outline a new model of care for stroke patients that can be delivered and tested in future research. They convincingly argue that providing integrated care in this area will improve care outcomes. Their paper contributes the essential initial modelling that enables programme commissioners to establish integrated care solutions to longstanding problems when providing care to stroke patients.

Serena Yu et al.'s article follows on from the previous one and makes the case for expanding integrated care provision in a fee for service primary care service in Australia. Their paper explores the costs and gains of implementing a new model of care within the primary care setting with expected attendant problems such as ensuring that general practitioners are sufficiently engaged in the process and committed to the new model. The authors conclude that implementing a new model requires considerable efforts to involve general practitioners without which the new care model would not be successful.

Barbara Murphy et al's tackle a related issue in care integration. Their paper investigates whether or not face-to-face network meetings increase the collaborative attitudes amongst medical staff in a mental health network in Australia. At the core, their paper analyses the 
contribution the network platform makes to the diffusion of practice, in this specific case, of collaborative working. Their findings are encouraging and highlight a critical aspect of any change in organisations, how to increase diffusion of new practices across professional groups.

In the next article by Darryl Phillipowsky, the focus shifts to the perceptions of social workers in integrated care settings. The author's findings strengthen the existing evidence on how professionals in integrated care settings see themselves working within different professional cultures. Phillipowsky contributes a critical perspective by probing the impact austerity had on the perception of roles and status of social workers in integration programmes. His paper adds an important piece in the puzzle of the context dependability of integration and illustrates the need for more research around the cost effectiveness of integration in times of decreasing health care budgets in real terms.

How critical the perceptions of professionals are to the success of integration projects has long been known. What is less known is how to effectively influence stakeholders engaged in integration such that they support the programme aims and objectives. A key objective in any integration project is to ensure that everyone works towards a set of shared goals. Cheung et al.'s article introduces readers to an exciting new tool to bring about a harmonisation of views amongst programme stakeholders. The paper argues that serious gaming may well have the potential to allow the participants in integration programmes to make their aims consistent and compatible with wider programme aims.

On a practice level, the article by Mette Kollerup et al.'s raises the important question as to what integration can contribute to the safe administration of medication to patients postdischarge. Nurse-led medication management is gaining attention as pressure on accidents and emergency departments increase and preventing re-admittance of patients through medication errors becomes paramount. Kollerup et al. build their case around the argument that improving patient safety requires a strong evidential link between integrated care and medication management and that this represents the next frontier in care management by nurses. The authors support the case for integrated care in this field by pointing to the detailed knowledge necessary for nurses to manage complex medications to patients postdischarge. Their article usefully opens up this field to further research to explore the impact care integration can have on medication administration and management.

This brings us to the last but one paper in this issue dealing with the fascinating issue of the drivers of change. There has been increased emphasis in the UK and other countries on leadership within health care and Steve Iliffe and Jill Manthorpe examine the role medical leadership plays in bringing about integration. This is not just a timely investigation but also one that recognises important current developments in health organisation where medical professionals are increasingly taking on management roles replacing or complementing health care managers. The authors present some encouraging evidence as to the positive effects of medical leaders in accelerating integration in the field and thus make a convincing case for more evidence in this area.

The issue is rounded up by a paper on co-production in health and social care integration by Kaehne, Beacham and Feather. The authors formulate a pragmatic approach and contrast this approach with a more idealistic notion of co-production. They set out some of the principles that govern a pragmatic approach and argue the case for a model of co-production that takes account of the huge diversity of programmes in the integration arena. The notion of diversity that animates their pragmatic approach also neatly takes the argument full circle to our starting point. Where integration in health and social care expands its reach into hitherto untouched fields and disciplines, the overall impression of integration practices is one of variability and contingency. It is up to us to make sense of it and create the systematic reservoir of knowledge that helps us identify best practice. 